\title{
MADNESS: A MULTIRESOLUTION, ADAPTIVE NUMERICAL ENVIRONMENT FOR SCIENTIFIC SIMULATION *
}

\author{
ROBERT J. HARRISON ${ }^{\dagger}$, GREGORY BEYLKIN ${ }^{\ddagger}$, FLORIAN A. BISCHOFF§, JUSTUS A. \\ CALVIN $『$, GEORGE I. FANN", JACOB FOSSO-TANDE** DIEGO GALINDO॥, JEFF R. \\ HAMMOND $^{\dagger \dagger}$ REBECCA HARTMAN-BAKER $\ddagger \ddagger$ JUDITH C. HILL"l, JUN JIA $\S$, JAKOB S. \\ KOTTMANN ${ }^{\S}$, M-J. YVONNE OU $₫$, LAURA E. RATCLIFF***MATTHEW G. REUTER ${ }^{\dagger}$,

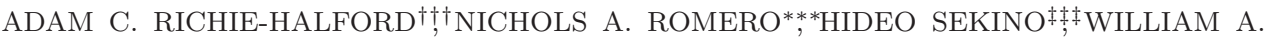 \\ SHELTON $\$ \S \S$ BRYAN E. SUNDAHL ${ }^{\dagger}$, W. SCOTT THORNTON ${ }^{\dagger}$, EDWARD F. VALEEV $^{\uparrow}$, \\ ÁLVARO VÁZQUEZ-MAYAGOITIA***NICHOLAS VENCE
}

Key words. 65-01; 65Y05; 65Z05; 65E05; 65N99; 65T60; 00A72; 81-08

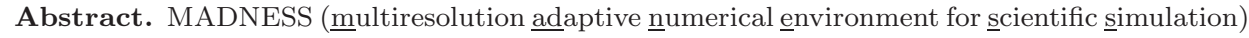
is a high-level software environment for solving integral and differential equations in many dimensions that uses adaptive and fast harmonic analysis methods with guaranteed precision based on multiresolution analysis and separated representations. Underpinning the numerical capabilities is a powerful petascale parallel programming environment that aims to increase both programmer productivity and code scalability. This paper describes the features and capabilities of MADNESS and briefly discusses some current applications in chemistry and several areas of physics.
\end{abstract}

1. Introduction. Numerical tools are ubiquitous in modern science since the relevant models/equations do not generally have analytical solutions. Although advances in scientific computing over the last thirty years have enabled more sophisticated models and the quantitative simulation of large-scale problems, these numerical methods introduce new, unphysical considerations that must also be taken into account. For instance, function representation, that is, the choice of basis set, is critically

*Corresponding author: Robert J. Harrison (robert.harrison@stonybrook.edu). This research was sponsored in part by the Office of Advanced Scientific Computing Research of the U.S. Department of Energy, and includes work performed at the Oak Ridge National Laboratory, which is managed by UT-Battelle, LLC under Contract No. DE-AC05-00OR22725. This work employed resources of the National Center for Computational Sciences at Oak Ridge National Laboratory and of the Argonne Leadership Computing Facility, which is a DOE Office of Science User Facility supported under Contract DE-AC02-06CH11357. R.J.H. was supported in part by the National Science Foundation under grant ACI-1450344.

†Stony Brook University, Stony Brook, New York 11794, USA

¥University of Colorado at Boulder, Boulder, Colorado 80309, USA

§Institut für Chemie, Humboldt-Universität zu Berlin, Unter den Linden 6, 10099 Berlin, Germany

ๆ Virginia Tech, Blacksburg, Virginia 24061, USA

" Oak Ridge National Laboratory, Oak Ridge, Tennessee 37831, USA

**Department of Chemistry and Biochemistry, Florida State University, Tallahassee, Florida 32306, USA

$\dagger^{\dagger}$ Parallel Computing Lab, Intel Corporation, Portland, Oregon 97219, USA

¥ National Energy Research Scientific Computing Center, Lawrence Berkeley National Laboratory, Berkeley, California 94720, USA

$\S \S$ LinkedIn Corporation, Mountain View, California 94043, USA

ฯ Department of Mathematical Sciences, University of Delaware, Newark, Delaware 19716, USA

*** Argonne Leadership Computing Facility, Argonne National Laboratory, Argonne, Illinois 60439, USA

$\dagger^{\dagger \dagger}$ Department of Physics, University of Washington, Seattle, Washington 98195, USA

$\ddagger \ddagger$ Computer Science and Engineering, Toyohashi University of Technology, Toyohashi, AICHI 441-8580, Japan

$\S \S$ Louisiana State University, Baton Rouge, Louisiana 70803, USA

ฯ Department of Physics, LaSierra University, Riverside, California 92505, USA 
important. If a basis set does not adequately resolve the finest details of the system, the calculated solutions will likely be inaccurate and have questionable physical interpretation. Other numerical factors include computational efficiency, scalability, distributed memory management, differentiation schemes, and quadrature rules. As such, most computational approaches force the scientist or engineer to compose software in terms of details inherent to the representation (e.g., the coefficients of basis functions or integrals within the basis set), rather than at the level of mathematics or physics that uses the natural functions or operators of the problem domain. In the end, all of these considerations obscure the desired science and force scientists to instead focus on computational mundanities.

Several software packages have been developed to help insulate scientists from these issues; two notable examples are Trilinos [28] and PETSc [9]. In essence, these and similar frameworks support common scientific computing operations, e.g., linear algebra algorithms, mesh generation (function representation), nonlinear solvers, and ordinary differential equation integrators. These packages also typically scale to parallel or massively-parallel machines, facilitating the solution of large-scale problems. Unfortunately, to our knowledge, all of these packages require the scientist to "think" in vectors and matrices, instead of functions and operators. Moreover, with an emphasis on engineering applications, computation is typically restricted to three or fewer dimensions.

Our goal in this communication is to motivate and overview the MADNESS (multiresolution adaptive numerical environment for s scientific simulation) package, which offers the following capabilities to users:

1. The illusion of basis set-free computation. Under this illusion, the user computes with functions and operators instead of vectors and matrices. Behind the scenes, functions and operators are expanded in a multi-wavelet basis, where the number of basis functions is adapted to guarantee the precision of each operation. As necessary, the "mesh", which is the support of the basis functions, is refined in computationallytroublesome regions (perhaps those with fine details) and coarsened in others, enabling the simulation of multi-scale problems.

2. Fast and arbitrarily-accurate methods for solving differential and integral equations in one to six dimensions (perhaps more), with specified boundary conditions. These operations include, for example, linear algebra, numerical differentiation/integration, and, perhaps most important, integral convolution kernels, such as the Poisson equation Green's function.

3. A parallel runtime that allows the user to compose algorithms as a dynamically scheduled set of tasks that operate on objects in global namespaces.

4. Algorithms and implementations that scale to massively-parallel computational resources, thereby enabling the solution of large problems.

We aim, by providing these tools, to raise the level of composition of scientific applications, making it faster and easier to both (i) construct robust and correct algorithms and (ii) calculate solutions to new and existing problems. Although MADNESS's breakthrough initial application was in computational chemistry [26, 50, 51], its framework more generally represents functions, operators and boundary conditions. Thus, it is readily applicable to describe and solve well-defined equations and boundary conditions. Extensive applications include, to date, atomic and molecular physics, electrostatics, materials science, nanoscience, nuclear physics, solvation models, and graph theory $[21,22,30,39,40,46,49]$. We hope that this introduction to MADNESS conveys its generality and encourages its application to new domains. 
The layout of this communication is as follows. Section 2 introduces the basics of multiresolution analysis (MRA), which is the mathematical underpinning of MADNESS, and how MRA facilitates scientific computing. We then provide and discuss, in $\S 3$, some simple examples of programming in MADNESS that illustrate the absence of basis set considerations and the ability to write code in terms of functions and operators. Then, $\S 4$ summarizes the computational details of MADNESS, including the runtime environment/framework and the methods behind MADNESS's utilization of (massively-)parallel architectures. Finally, §5 summarizes this report and describes future directions for MADNESS.

2. Numerics: Mathematical Overview \& Capabilities. With the illusion of basis free computation, a MADNESS user simply needs to specify the desired accuracy; from this, the numerical framework provides fast computation with guaranteed accuracy (in up to 6 dimensions). This is accomplished through several key mechanisms:

1. The underlying representation is an orthonormal basis [7]; functions are represented on an adaptive mesh with a discontinuous spectral-element basis set. Within each adaptively-refined element the basis functions are tensor products of Legendre polynomials. Crucially, the application programmer only uses the function as a single entity and never sees or manipulates the basis or mesh.

2. Guaranteed accuracy for each operation (e.g., multiplication or addition of functions, application of an operator) is accomplished through automatic refinement of the mesh using criteria derived from MRA [7].

3. Fast and accurate computation is enabled through MRA that separates behavior between length scales. MRA provides a sparse hierarchical (tree) decomposition of functions and operators that enables automatic refinement or coarsening of the mesh to meet the accuracy goal. MRA also yields fast algorithms for physically important operators $[7,12,20]$.

4. Fast and accurate computation beyond one dimension is made feasible through accurate and compact representations of operators as sums of separable kernels (usually Gaussians), which are created through quadrature or more advanced numerical techniques $[12,13,25]$.

In the remainder of this section, we elaborate on these points.

2.1. Underlying basis and adaptive mesh. Inside MADNESS (using a 1D example here, for simplicity), the user's computational domain is mapped to $[0,1]$. This domain is repeatedly subdivided by factors of two such that, at level $n$, there are $2^{n}$ boxes, each of size $2^{-n}$. Within each box, we use the first $k$ Legendre polynomials as the basis (or scaling functions in the language of MRA $[6,7]$ ). The polynomials are scaled and shifted to form an orthonormal basis.

Specifically, the $i^{\text {th }}$ scaling function $(i=0, \ldots, k-1)$ in the $l^{\text {th }}$ box $\left(l=0, \ldots, 2^{n}-\right.$ $1)$ at level $n(n=0,1, \ldots)$ is

$$
\phi_{l i}^{n}(x)=2^{n / 2} \phi_{i}\left(2^{n} x-l\right),
$$

where

$$
\phi_{i}(x)=\left\{\begin{array}{rr}
\sqrt{2 i+1} P_{i}(2 x-1), & x \in(0,1) \\
0, & x \notin(0,1)
\end{array}\right.
$$

is the $i^{\text {th }}$ mother scaling function. Projection of a function $f(x)$ into the basis at level 
$n$ is accomplished by orthogonal projection,

$$
\begin{aligned}
f^{n}(x) & =\sum_{l i} s_{l i}^{n} \phi_{l i}^{n}(x) \\
s_{l i}^{n} & =\int \mathrm{d} x f(x) \phi_{l i}^{n}(x)
\end{aligned}
$$

Projection is not performed globally on a uniform fine grid; instead, projection starts on a relatively coarse level $(n)$. Within each box on level $n$ we compute the projection at level $n$ and at the coarser level $n-1$. If the difference between these projections is not satisfactory, the projection is locally repeated at a finer level $(n+1)$, and so forth until the projection is sufficiently accurate. The differences in representations between successive levels are efficiently and accurately computed in the wavelet basis, which we now describe.

2.2. Multiresolution analysis. Rather than thinking about the approximation of a function at each length scale, MRA focuses upon the difference between length scales and, by doing so, exploits both smoothness and sparsity for fast computation. The telescoping series clearly illustrates this. Let $V^{n}$ be the space spanned by the polynomials at level $n$. Then, we can write

$$
V^{n}=V^{0} \oplus\left(V^{1} \ominus V^{0}\right) \oplus\left(V^{2} \ominus V^{1}\right) \oplus \cdots \oplus\left(V^{n} \ominus V^{n-1}\right)
$$

which decomposes the approximation space at level $n$ in terms of the coarsest approximation $\left(V^{0}\right)$ plus corrections at successive levels of refinement. If the function of interest is sufficiently smooth in some volume, then differences at finer scales will be negligible in that volume. Thus, smoothness is translated into sparsity and hence fast computation; a precise definition of "negligible" enables adaptive refinement to guarantee accuracy. The multi-wavelets at level $n$ are defined as an orthonormal, disjoint basis that spans the difference space $W^{n}=V^{n+1} \ominus V^{n}$ and are constructed by translation and dilation of the mother wavelets $[6,7]$.

We thus have two representations of our function: (i) its projection onto the finest level of the adaptively refined grid (i.e., in real space or in the scaling function basis) and (ii) its representation at the coarsest level plus corrections at successive levels of refinement (i.e., in the multi-wavelet basis). The fast wavelet transform (FWT) [11] enables fast and accurate transformation between these two representations. From a practical perspective, we simply pick the most convenient basis to compute in, just as one would if using the Fourier basis and the fast Fourier transform. However, the disjoint support of multi-wavelets enables local adaptive refinement, which is not possible in the global Fourier basis. A third equivalent representation is the value of the function at the Gauss-Legendre quadrature points within each box of the mesh, which facilitates certain operations (such as multiplication of functions).

We can use a similar approach to understand how to turn smoothness into sparsity in applying operators. Let $P^{n}$ and $Q^{n}$ be the projectors onto $V^{n}$ and $W^{n}$, respectively. Then, by construction, $P^{n+1}=P^{n}+Q^{n}$. Let $T$ be the kernel of an operator we wish to apply and let us assume that level $n$ is adequate to resolve both the input and 
output functions. The representation of the operator at level $n$ is

$$
\begin{aligned}
T^{n} & =P^{n} T P^{n} \\
& =\left(P^{n-1}+Q^{n-1}\right) T\left(P^{n-1}+Q^{n-1}\right) \\
& =T^{n-1}+Q^{n-1} T Q^{n-1}+Q^{n-1} T P^{n-1}+P^{n-1} T Q^{n-1} \\
& =T^{0}+\sum_{m=0}^{n-1}\left(A^{m}+B^{m}+C^{m}\right),
\end{aligned}
$$

where we define $A^{m}=Q^{m} T Q^{m}, B^{m}=Q^{m} T P^{m}$, and $C^{m}=P^{m} T Q^{m}$. In this socalled non-standard form [10], $T^{0}$ acts only upon scaling functions at the coarsest level whereas the operators applied at finer levels all involve wavelets.

For a demonstrative example of the computational significance of this approach, let us examine convolution with the Poisson kernel $[7,25,26], T=|\vec{x}-\vec{y}|^{-1}$. Consider the operator $A^{m}$, which connects wavelets at level $m$ to other wavelets at level $m$. By construction, the first $k$ moments of the wavelets vanish; thus, the multipole expansion of $T$ tells us that the matrix elements of $A^{m}$ decay as $r^{-2 k-1}$ (e.g., if $k=8$, which is routine, they decay as $r^{-17}$ ). In other words, even though $T$ is dense, $A^{m}$ is very sparse to any finite precision. A similar approach shows that $B^{m}$ and $C^{m}$ decay as $r^{-k-1}$. Translationally invariant operators yield Toeplitz representations, which are very important for practical computation due to the greatly reduced burden of computing and storing the operator.

2.3. Separated representations. The approach described above is not efficient beyond one dimension because the cost of naïvely applying an operator grows as $O\left((2 k)^{2 D} \log \left(\epsilon^{-1}\right)^{D}\right)$ with similarly excessive memory requirements, where $D$ is the dimensionality and $\epsilon$ is the desired precision. However, for many physically important operators we can construct $[12,25,26]$ either global or local (via numerically generated low-rank factorizations of the full operator) separated representations; that is, we can approximate, with controlled precision, the full operator as a practically short sum of products of operators that separately apply in each dimension. This reduces the cost of application to $O\left(M(2 k)^{D+1} \log \left(\epsilon^{-1}\right)^{D}\right)$ and the memory footprint to $O\left(M D(2 k)^{2} \log \left(\epsilon^{-1}\right)\right)$ where $M$ is the number of terms in the sum. Powerful techniques for generating these separated forms include quadrature of integral identities [25] and numerical techniques developed by Beylkin and Monzón [13]. For example, numerical quadrature to evaluate the integral

$$
r^{-1}=\frac{2}{\sqrt{\pi}} \int_{-\infty}^{\infty} \mathrm{d} s e^{-r^{2} e^{2 s}+s}
$$

yields compact representations of $r^{-1}$ as a sum over Gaussians,

$$
=\sum_{\mu=1}^{M} c_{\mu} e^{-t_{\mu} r^{2}}+O\left(\epsilon r^{-1}\right) ;
$$

in which the number of terms $(M)$ scales only as $O\left((\log R)\left(\log \epsilon^{-1}\right)\right)$, where $[1, R]$ is the range of validity and $\epsilon$ is the relative precision.

3. Applications: Programming with MADNESS. Having discussed the mathematical underpinnings of MADNESS, we briefly examine some samples of numerical programming with MADNESS. Many additional examples, including quantum 
chemistry and other applications, are provided in the MADNESS code repository (see the directories src/apps/ and src/examples/ in the code [1]).

3.1. Example: Getting Started with MADNESS. Our first example aims to evaluate the integral (trace), 2-norm, and electrostatic self-energy of a Gaussian function in three dimensions.

$$
\begin{aligned}
g(\vec{x}) & =e^{-|\vec{x}|^{2}} \\
\int \mathrm{d}^{3} \vec{x} g(\vec{x}) & =\pi^{3 / 2} \doteq 5.5683279 \\
\left(\int \mathrm{d}^{3} \vec{x} g(\vec{x})^{2}\right)^{1 / 2} & =(\pi / 2)^{3 / 4} \doteq 1.403104 \\
\int \mathrm{d}^{3} \vec{x} g(\vec{x}) \int \mathrm{d}^{3} \vec{y}|\vec{x}-\vec{y}|^{-1} g(\vec{y}) & =\pi^{5 / 2} 2^{1 / 2} \doteq 24.739429
\end{aligned}
$$

Listing 1 shows the entire MADNESS source code for this task, which we now walk through.

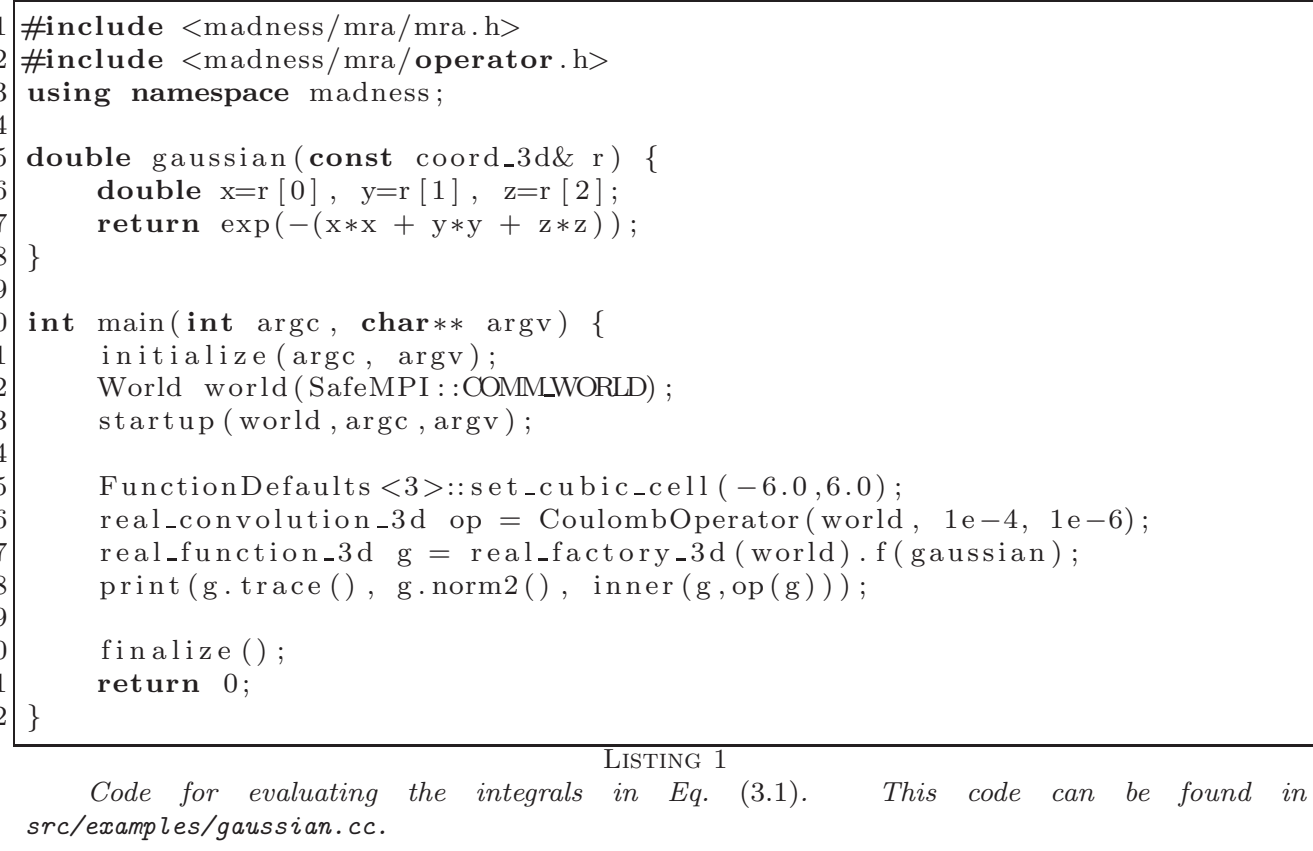

Code for evaluating the integrals in Eq. (3.1). This code can be found in src/examples/gaussian.cc.

Initially ignoring the boiler-plate code, there are only four lines of significance.

- Line 15 defines the computational volume $\left([-6,6]^{3}\right)$.

- Line 16 constructs the Coulomb operator; in actuality, a separated Gaussian representation of the Green's function for Poisson's equation with free-space boundary conditions, as described in $\S 2.3$. The two numerical values in the constructor indicate the finest length scale to resolve and the desired accuracy of the operator, respectively.

- Line 17 projects the analytic function (lines 5-8) into the adaptive numerical basis using the default precision $\left(\epsilon=10^{-4}\right)$ and wavelet order $(k=6)$.

- Line 18 prints the results.

The integral in (3.1d) (the last printed value) is computed as the inner product (inner) of the Gaussian function $(\mathrm{g})$ and its convolution with the Green's function (op $(\mathrm{g})$ ). 
Printed results agree with the exact values to six significant figures, which is more than might be expected from the default precision of $10^{-4}$. This is a common observation and is likely due to the projection into the numerical basis oversampling to guarantee the requested precision.

The main program commences (line 11) by initializing the MADNESS parallel computing environment (discussed in $\S 4$ ), which includes initializing MPI (if it has not yet been initialized). Next, line 12 creates a World object that encapsulates an MPI intra-communicator; this plays the same role as an MPI communicator, but provides the much more powerful capabilities of the MADNESS runtime. Subsequently, line 13 initializes the MADNESS numerical environment. The program concludes by terminating the parallel computing environment (line 20), which also finalizes MPI if the call to initialize() initialized MPI.

Finally, the MADNESS framework provides support for common mathematical and computational processes. For example, algorithms are implemented for (i) matrixfree iterative solvers (such as GMRES [41] and BiCGStab [48]), (ii) converting between the multi-wavelet basis and a Fourier basis [29], (iii) solving non-linear systems of equations [24], and (iv) outputting MADNESS functions for visualization with various tools, including VisIt [4], OpenDX [2], and ParaView [27].

3.2. Molecular electronic structure. One of the initial targets when developing MADNESS was quantum chemistry, where electronic structure problems for atoms and molecules are solved using the density functional theory (DFT) or the Hartree-Fock methods [25, 26, 51]. Either of these approaches requires solving a set of nonlinear partial differential equations, which, for time-independent systems, are typically cast into an eigenvalue problem,

$$
\left(-\frac{1}{2} \nabla^{2}+V(\vec{x})\right) \psi(\vec{x})=E \psi(\vec{x})
$$

$V(\vec{x})$ is the potential energy for an electron at position $\vec{x}$ and is itself dependent on $\psi(\vec{x})$ (thus the nonlinearity). The eigenvalues are interpreted as energies of molecular states and the eigenfunctions as molecular orbitals.

The standard approach for solving Eq. (3.2) expands $\psi(\vec{x})$ in a predefined, fixed set of basis functions, thereby converting Eq. (3.2) into a generalized matrix equation. Our MADNESS implementation follows a different approach [34] to utilize the computational strengths of MADNESS and to avoid the numerical problems associated with derivative operators on deeply refined meshes. We recast Eq. (3.2) as an integral equation,

$$
\psi(\vec{x})=-2 \int \mathrm{d} \vec{x}\left(-\nabla^{2}-2 E\right)^{-1} V(\vec{x}) \psi(\vec{x}),
$$

which can be solved as a fixed point iteration to the lowest eigenvalue. The integral kernel in Eq. (3.3) (which assumes free-space boundary conditions),

$$
G\left(\vec{x}, \vec{x}^{\prime}\right)=\frac{1}{4 \pi} \frac{e^{-\sqrt{-2 E}}}{\left|\vec{x}-\vec{x}^{\prime}\right|}
$$

not only incorporates the correct long-range asymptotic form for bound-state (i.e., $E<0)$ molecular orbitals, but it can be efficiently applied using the separated representations discussed in $\S 2.3$. Capabilities of the molecular electronic structure code include energies for a wide range of functionals (including hybrids) and for many-body 
methods (second-order Møller-Plesset in six dimensions [14, 15] and configuration interaction [35]), localized orbitals for reduced scaling, forces [50], solvation [22], and linear-response for excited states and dynamic polarizabilities [44, 52].

3.3. Nuclear physics. Nuclear physics is another successful application for MADNESS [21, 36, 37, 38]. Nuclear density functional theory (NDFT) extends the molecular DFT above to describe the complex superfluid many-fermion and boson system. A critical difference of NDFT is the use of two-component and four-component complex orbitals with particle spins and the appearance of resonance and continuum states. For example, the two-component complex wave-functions, $u$ and $v$, and an associated pairing potential, $\Delta$, extend the one-component orbital $(\psi)$ in the molecular DFT to NDFT. These modifications ultimately result in the Hartree-Fock-Bogoliubov (HFB) equations,

$$
\left[\begin{array}{cc}
h_{\uparrow}-\lambda_{\uparrow} & \Delta \\
\Delta^{*} & h_{\downarrow}-\lambda_{\downarrow}
\end{array}\right]\left[\begin{array}{l}
u_{i} \\
v_{i}
\end{array}\right]=E_{i}\left[\begin{array}{c}
u_{i} \\
v_{i}
\end{array}\right]
$$

where $h_{\uparrow \downarrow}$ is a single particle Hamiltonian for the given component and $\lambda_{\uparrow \downarrow}$ is the chemical potential for that component. For the ultracold fermionic gas in the BECBCS (Bose-Einstein condensate - Bardeen-Cooper-Schriffer) crossover simulation, the oscillatory Fulde-Ferrell-Larkin-Ovchinnikov phase was found to have oscillating pairing gaps such that

$$
h_{\uparrow \downarrow}=-\frac{1}{2} \nabla \cdot\left[\nabla \alpha_{\uparrow \downarrow}(r)\right]+V_{\uparrow \downarrow}(r) .
$$

$\alpha_{\uparrow \downarrow}$ is the kinetic energy density and $V_{\uparrow \downarrow}$ is the trapping potential. The HFB equations are solved with a complex version of the scheme used by the molecular density functional application with additional geometric constraints; Eq. (3.5) is transformed into a coupled set of integral equations, to which Eq. (3.4) is applied.

3.4. Atomic and molecular physics. The above applications are all timeindependent (or introduce time-dependence only through response theory). In [49] MADNESS was used by physicists interested in the effects of non-perturbative, fewcycle laser pulses on atoms and molecules. This research prompted us to develop numerically robust and accurate techniques for evolving quantum wavepackets (and more general systems of partial differential and integral equations) forward in time. The familiar challenges of time evolution are exacerbated by adaptive refinement and truncation of the multi-wavelet basis. Basis truncation introduces high-frequency noise, as do the inevitable discontinuities between adjacent sub-domains. This noise must be removed from the simulation for accuracy and efficiency. Unfortunately, we cannot casually accept that frequencies beyond some band limit (cut-off) will be propagated inaccurately; for example, the wave function of the time-dependent Schrödinger equation (TDSE) depends upon accurate phase information. Our solution was to choose a band limit, propagate exactly below the band limit, and filter frequencies above it - essentially as if we were computing on a globally very fine, uniform mesh but still retaining the advantages of adaptive refinement.

The TDSE was solved [49] for hydrogenic atoms and other systems subjected to a strong, attosecond laser field, and the photoionization cross section and other observables were computed. The electron behavior was modeled by a three-dimensional wave function, $\Psi(\vec{x}, t)$, which, within the dipole approximation and in atomic units, 


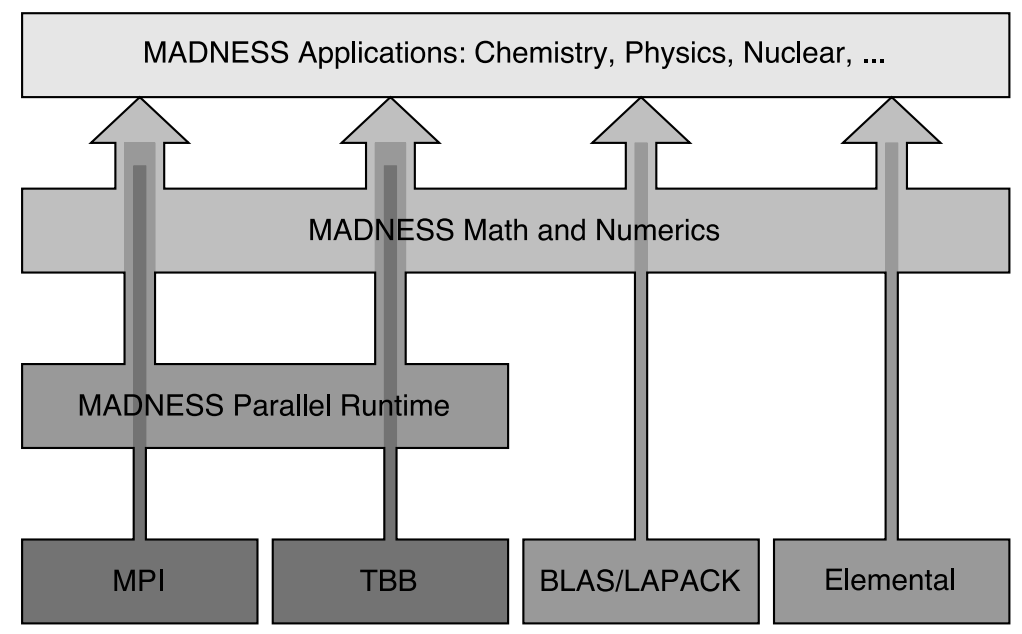

FIG. 4.1. The library structure of MADNESS and its dependencies. Each of these sub components has its own public application programing interface (API) that may be used both for development inside MADNESS as well as in external software packages.

evolves according to the TDSE,

$$
i \frac{d \Psi(\vec{x}, t)}{d t}=\left(-\frac{1}{2} \nabla^{2}+V(\vec{x})+\vec{E}(t) \cdot \vec{x}\right) \Psi(\vec{x}, t),
$$

where $\vec{E}$ is the electric field and $V$ the atomic potential. The initial state, $\Psi(\vec{x}, 0)$, is the ground state; that is, the solution of Eq. (3.2) with the smallest $E$.

The most efficient evolution was performed with a fourth-order accurate, gradientcorrected exponential propagator [19] that is only slightly more expensive than the second-order accurate Trotter approximation. Crucial for efficient and accurate application was the realization that, while the kernel of the free-space propagator,

$$
(2 \pi i t)^{-1 / 2} \exp \left(-|\vec{x}|^{2} / 2 i t\right),
$$

is infinite in extent and highly oscillatory, its combination with a projector onto a finite bandlimit is compact and also bandlimited.

4. Computational Framework: The MADNESS Parallel Runtime. The MADNESS parallel runtime, like the numerical libraries of MADNESS, is an intuitive interface that allows the user to compose algorithms as a dynamically scheduled set of tasks that operate on objects in global namespaces. This high-level approach to parallel programming offers greater composability than that of the traditional Message Passing Interface (MPI) and explicit thread programming (POSIX or $\mathrm{C}++$ threads). The key runtime features include the use of

1. global namespaces for building applications that are composed of (global) objects interacting via remote methods;

2. tasks as first-class entities for fine-grained work decomposition;

3. futures [8] for expressing dependencies between the tasks.

These features permit the programmer to deal less with the explicit low-level details of computation and data partitioning (e.g., message passing between processes with disjoint data or threads operating on shared data) and focus more on high-level concepts central to the scientific domain of interest. An algorithm properly composed 
within the MADNESS runtime will (partially) hide costs of data communication and be tolerant to load imbalance due to dynamic, data-driven work scheduling.

Many of the individual concepts appear in other packages, for example, in Cilk $[16,17]$, Charm $++[31,32,33]$, Intel Threading Building Blocks (TBB) [3], and other projects including ACE [42, 43] and PGAS languages [53]. Some of these features are starting to make their way into mainstream programming languages. $\mathrm{C}++$, for example, has included task-based concurrency with asynchronous function calls and futures since 2011 (note that MADNESS futures, unlike C++ futures, can refer not only to results of local tasks, but also to those of remote tasks).

For the sake of portability the MADNESS parallel runtime is implemented in $\mathrm{C}++$ (2011 standard), MPI-1 and POSIX threads; all non-portable software prerequisites, such as Intel TBB, are optional. In the most common scenario, each node executes a single MPI process composed of the main application thread. The MADNESS runtime spawns a remote method invocation (RMI) thread and a pool of computational threads managed by a task queue; these threads are not directly accessible to the user. The main application thread runs sequentially through the main program and submits tasks (that can themselves generate more tasks) to the local and/or remote task queue. ${ }^{1}$ The output of each task is encapsulated in a future that can serve as input to other tasks. Once its input futures are available (assigned), the task queue schedules the tasks for execution. Because MADNESS futures live in a global namespace, assigning a future can involve data movement to a remote process; this process is facilitated by the RMI thread in the receiving process. When available, MADNESS can use the task queue provided by the Intel TBB library; MADNESS defaults to its own task queue implemented in terms of POSIX threads.

By supporting the straightforward composition of a distributed task-based application, the MADNESS runtime allows programmers to focus on exposing concurrency by decomposing data and computation and/or by optimizing load balance. The lowlevel details of thread scheduling and synchronization, data movement, and communication hiding are automated. However, the MADNESS runtime provides access to enough low-level details of the architecture (e.g., process rank) that the placement of data and computation can be directly controlled by the user. This allows tight orchestration of algorithms with complex data flows, such as in the systolic loops used for computing localized electronic states in molecular electronic structure. These abilities, as provided by the MADNESS runtime, are also used by TiledArray [18] (a framework for block-sparse tensor computations) to hide communication costs and withstand load imbalances in handling block-sparse data.

In addition to the above core capabilities, the MADNESS parallel runtime also provides higher-level constructs built on these features. One such feature is a distributedmemory, associative container used to store the functions and operators in the MADNESS numerical library. This container automates the placement and computation of distributed data. Other features include parallel algorithms (e.g., parallel for-each) and task-based collective operations. Many of these components interoperate via futures and, therefore, fit naturally within the task-based framework. For example, the task-based all-reduce algorithm, which is analogous to the MPI_Allreduce function, uses futures as both the input and output of the function, allowing a seamless connection between local computation tasks, collective communication, and remotecomputation tasks.

\footnotetext{
${ }^{1}$ The main thread can also act as a computational thread by executing tasks in the task queue while waiting for an event (e.g., while waiting inside a barrier).
} 
5. Summary. Since the introduction and initial successful science application of MADNESS, it received an R\&D 100 Award in 2011 [5], and there have been at least three independent implementations of the associated numerical methods in Japan [45], Norway [23], and the United States [47]. MADNESS itself is now thriving as an open-source, community project with financial support from multiple sources. Central to people's interest is the emphasis on a high-level of composition while maintaining guarantees of accuracy and speed. It is not that MADNESS is necessarily the fastest code for any particular problem, but rather that a user working on tractable problems will get the right answer with modest effort and without having to place unnecessary emphasis on computational details. However, the caveat of "tractability" is nontrivial - user attention is still needed to regularize some singularities, and the fast algorithms within MADNESS (notably the integral convolution kernels) do not yet include scattering operators, which is a subject of current research. Similarly, efficient computation is presently limited to simple domains and, as shown in [39], cumbersome techniques are presently necessary to accommodate even simple interior boundary conditions. Nevertheless, the numerical techniques have demonstrated their worth in a broad range of physically interesting applications. Similarly, the MADNESS parallel runtime is being successfuly used for petascale computations independent of the numerical layer [18], illustrating the power and utility of the massively threaded, task-based approach to computation.

\section{REFERENCES}

[1] https://github.com/m-a-d-n-e-s-s/madness.

[2] OpenDX, http://www.opendx.org.

[3] Threading Building Blocks, https://www.threadingbuildingblocks.org.

[4] VisIt, https://wci.llnl.gov/simulation/computer-codes/visit.

[5] Free framework for scientific simulation, R\&D Magazine, 53 (2011), p. 28.

[6] B. Alpert, A class of bases in $L^{2}$ for the sparse representation of integral operators, SIAM J. Math. Anal., 24 (1993), pp. 246-262.

[7] B. Alpert, G. Beylkin, D. Gines, and L. Vozovoi, Adaptive Solution of Partial Differential Equations in Multiwavelet Bases, J. Comput. Phys., 182 (2002), pp. 149-190.

[8] Henry G. BAKER JR. AND CARL HewitT, The incremental garbage collection of processes, in Proceedings of the 1977 Symposium on Artificial Intelligence and Programming Languages, ACM, New York, NY, USA, 1977, pp. 55-59.

[9] Satish Balay, Jed Brown, Kris Buschelman, Victor Eijkhout, William D. Gropp, Dinesh Kaushik, Matthew G. Knepley, Lois Curfman McInnes, Barry F. Smith, and Hong Zhang, PETSc Users Manual, tech. report, Argonne National Laboratory, 2011.

[10] Gregory Beylkin, Vani Cheruvu, and Fernando PÉrez, Fast adaptive algorithms in the non-standard form for multidimensional problems, Appl. Comput. Harmon. Anal., 24 (2008), pp. 354-377.

[11] G. Beylkin, R. Coifman, and V. Rokhlin, Fast wavelet transforms and numerical algorithms I, Comm. Pure Appl. Math., 44 (1991), pp. 141-183.

[12] Gregory Beylkin, Robert Cramer, George Fann, and Robert J. Harrison, Multiresolution separated representations of singular and weakly singular operators, Appl. Comput. Harmon. Anal., 23 (2007), pp. 235-253.

[13] Gregory Beylkin and Lucas Monzón, Approximation by exponential sums revisited, Appl. Comput. Harmon. Anal., 28 (2010), pp. 131-149.

[14] Florian A. Bischoff, Robert J. Harrison, and Edward F. Valeev, Computing ManyBody Wave Functions with Guaranteed Precision: The First-Order Møller-Plesset Wave Function for the Ground State of Helium Atom, J. Chem. Phys., 137 (2012), p. 104103.

[15] Florian A. Bischoff And Edward F. VAleev, Computing molecular correlation energies with guaranteed precision, J. Chem. Phys., 139 (2013), p. 114106.

[16] Robert D. Blumofe, Christopher F. Joerg, Bradley C. Kuszmaul, Charles E. Leiserson, Keith H. Randall, and Yuli Zhou, Cilk: An efficient multithreaded runtime system, in Proceedings of the Fifth ACM SIGPLAN Symposium on Principles and Prac- 
tice of Parallel Programming (PPoPP), Santa Barbara, California, 7 1995, pp. 207-216.

[17] R. D. Blumofe, C. F. Joerg, B. C. Kuszmaul, C. E. Leiserson, K. H. Randall, and Y. L. ZHOU, Cilk: An efficient multithreaded runtime system, J. Parallel Distrib. Comput., 37 (1996), pp. 55-69.

[18] Justus A. Calvin and Edward F. Valeev, Task-based algorithm for matrix multiplication: A step towards block-sparse tensor computing, 2015. arXiv:1504.05046.

[19] Siu A. Chin And C. R. Chen, Fourth order gradient symplectic integrator methods for solving the time-dependent schrödinger equation, J. Chem. Phys., 114 (2001), pp. 7338-7341.

[20] G. Fann, G. Beylkin, R. J. Harrison, and K. E. Jordan, Singular Operators in Multiwavelet Bases, IBM J. Res. Devel., 48 (2004), pp. 161-171.

[21] G. I. Fann, J. Pei, R. J. Harrison, J. Jia, J. Hill, M. Ou, W. Nazarewicz, W. A. Shelton, AND N. Schunck, Fast multiresolution methods for density functional theory in nuclear physics, J. Phys.: Conf. Ser., 180 (2009), p. 012080.

[22] Jacob Fosso-TANde AND RoBert J. HARRISOn, Implicit solvation models in a multiresolution multiwavelet basis, Chem. Phys. Lett., 561-562 (2013), pp. 179-184.

[23] Luca Frediani, Eirik Fossgand, Tor Fla, and Kenneth Ruud, Fully Adaptive Algorithms for Multivariate Integral Equations using the Non-Standard Form and Multiwavelets with Applications to the Poisson and Bound-State Helmholtz Kernels in Three Dimensions, Mol. Phys., 111 (2013), pp. 1143-1160.

[24] Robert J. Harrison, Krylov subspace accelerated inexact newton method for linear and nonlinear equations, J. Comput. Chem., 25 (2004), pp. 328-334.

[25] R. J. Harrison, G. I. Fann, Takeshi Yanai, and G. Beylkin, Multiresolution quantum chemistry in multiwavelet bases, in Lecture Notes in Computer Science, vol. 2660, SpringerVerlag, Heidelberg, 2003, pp. 103-110.

[26] Robert J. Harrison, George I. Fann, Takeshi Yanai, Zhengting Gan, and Gregory BEYLKIN, Multiresolution quantum chemistry: Basic theory and initial applications, J. Chem. Phys., 121 (2004), pp. 11587-11598.

[27] A. Henderson, ParaView Guide, A Parallel Visualization Application, Kitware Inc., 2007.

[28] Michael A. Heroux, Roscoe A. Bartlett, Vicki E. Howle, Robert J. Hoekstra, Jonathan J. Hu, Tamara G. Kolda, Richard B. Lehoucq, Kevin R. Long, Roger P. Pawlowski, Eric T. Phipps, Andrew G. Salinger, Heidi K. Thornquist, Ray S. Tuminaro, James M. Willenbring, Alan Williams, and Kendall S. Stanley, An overview of the trilinos project, ACM Trans. Math. Softw., 31 (2005), pp. 397-423.

[29] Jun Jia, Robert Harrison, and George Fann, Fast transform from an adaptive multiwavelet representation to a partial Fourier representation, J. Comput. Phys., 229 (2010), pp. 5870-5878.

[30] J. Jia, J. C. Hill, G. I. FAnn, AND R. J. HARRISOn, Multiresolution fast methods for a periodic 3-d Navier-Stokes solver, in DCABES 2009: The 8th International Symposium on Distributed Computing and Applications to Business, Engineering, and Science, 2009, pp. $13-16$.

[31] L. V. Kalé, Parallel programming with CHARM: An overview, tech. report, Parallel Programming Laboratory, University of Illinois at Urbana-Champaign, July 1993.

[32] Laxmikant V. Kalé and Sanjeev Krishnan, CHARM++: A Portable Concurrent Object Oriented System Based on $\mathrm{C}++$, in Proceedings of the Eighth Annual Conference on Object-oriented Programming Systems, Languages, and Applications, Anrdeas Paepcke, ed., ACM Press, September 1993, pp. 91-108.

[33] L. V. Kalé, B. Ramkumar, A. B. Sinha, and V. A. Saletore, The Charm Parallel Programming Language and System: Part II - The Runtime system, tech. report, Parallel Programming Laboratory, University of Illinois at Urbana-Champaign, 1994.

[34] M. H. Kalos, Monte Carlo Calculations of the Ground State of Three- and Four-Body Nuclei, Phys. Rev., 128 (1962), pp. 1791-1795.

[35] Jakob S. Kottmann, Sebastian Höfener, and Florian A. Bischoff, Numerically accurate linear response-properties in the configuration-interaction singles (CIS) approximation, Phys. Chem. Chem. Phys., (2015), p. DOI:10.1039/C5CP00345H.

[36] J. C. Pei, G. I. Fann, R. J. Harrison, W. Nazarewicz, J. Hill, D. Galindo, and J. Jia, Coordinate-Space Hartree-Fock-Bogoliubov for Superfluid Fermi Systems in Large Boxes, J. Phys. Conf. Ser., 402 (2012), p. 012035.

[37] J. C. Pei, G. I. Fann, R. J. Harrison, W. Nazarewicz, Y. Shi, and S. Thornton, Adaptive multi-resolution 3D Hartree-Fock-Bogoliubov solver for nuclear structure, Phys. Rev. C, 90 (2014), p. 024317.

[38] J. C. Pei, M. V. Stoitsov, G. I. Fann, W. Nazarewicz, N. Schunck, and F. R. Xu, Deformed coordinate-space Hartree-Fock-Bogoliubov approach to weakly bound nuclei and 
large deformations, Phy. Rev. C, 78 (2008), p. 064306.

[39] Matthew G. Reuter, Judith C. Hill, and Robert J. Harrison, Solving PDEs in irregular geometries with multiresolution methods I: Embedded Dirichlet boundary conditions, Comput. Phys. Commun., 183 (2012), pp. 1-7.

[40] Matthew G. Reuter, Mark A. Ratner, and Tamar Seideman, Laser alignment as a route to ultrafast control of electron transport, Phys. Rev. A, 86 (2012), p. 013426.

[41] Youcef SaAd and Martin H. Schultz, GMRES: A Generalized Minimal Residual Algorithm for Solving Nonsymmetric Linear Systems, SIAM J. Sci. Stat. Comput., 7 (1986), pp. 856869.

[42] Douglas C. Schmidt, The ADAPTIVE Communication Environment (ACE).

[43] Douglas C. Schmidt and Stephen D. Huston, C++ Network Programming: Systematic Reuse with ACE and Frameworks, Vol. 2, Pearson Education, 2002.

[44] H. Sekino, Y. Maeda, T. Yanai, And R. J. Harrison, Basis set limit hartree-fock and density functional theory response property evaluation by multiresolution multiwavelet basis, J. Chem. Phys., 129 (2008), p. 034111.

[45] H. Sekino, T. Oкамото, And S. Hamada, Solution of wave-equation using multiresolution multiwavelet basis function, in 2010 International Conference on Wavelet Analysis \& Pattern Recognition (ICWAPR), 2010, pp. 355-358.

[46] B.D. Sullivan, D. Weerapurage, And C. Groer, Parallel algorithms for graph optimization using tree decompositions, in Parallel and Distributed Processing Symposium Workshops PhD Forum (IPDPSW), 2013 IEEE 27th International, May 2013, pp. 1838-1847.

[47] E. F. Valeev and F. A. Bischoff, Private communication, 2011.

[48] H. A. van Der Vorst, Bi-CGSTAB: A Fast and Smoothly Converging Variant of Bi-CG for the Solution of Nonsymmetric Linear Systems, SIAM J. Sci. Stat. Comput., 13 (1992), pp. 631-644.

[49] Nicholas Vence, Robert Harrison, and Predrag Krstić, Attosecond electron dynamics: A multiresolution approach, Phys. Rev. A, 85 (2012), p. 033403.

[50] Takeshi Yanai, George I. Fann, Zhengting Gan, Robert J. Harrison, and Gregory BEYLKIN, Multiresolution quantum chemistry in multiwavelet bases: Analytic derivatives for Hartree-Fock and density functional theory, J. Chem. Phys., 121 (2004), pp. 2866-2876.

[51] Takeshi Yanai, George I. Fann, Zhenting Gan, Robert J. Harrison, and Gregory BEYLKin, Multiresolution quantum chemistry in multiwavelet bases: Hartree-Fock exchange, J. Chem. Phys., 121 (2004), pp. 6680-6688.

[52] Takeshi Yanai, Robert J. Harrison, and Nicholas C. Handy, Multiresolution quantum chemistry in multiwavelet bases: time-dependent density functional theory with asymptotically corrected potentials in local density and generalized gradient approximations, Mol. Phys, 103 (2005), pp. 413-424.

[53] Katherine Yelick, Dan Bonachea, Wei-Yu Chen, Phillip Colella, Kaushik Datta, Jason Duell, Susan L Graham, Paul Hargrove, Paul Hilfinger, Parry Husbands, ET AL., Productivity and performance using partitioned global address space languages, in Proceedings of the 2007 international workshop on Parallel symbolic computation, ACM, 2007, pp. 24-32. 